\title{
VARIACIÓN DEL PATRÓN DE ESCUTELACIÓN NUCAL DEL COCODRILO AMERICANO (CROCODYLUS ACUTUS CUVIER 1807) EN LA VENTANILLA, OAXACA, MÉXICO
}

\author{
Jesús García-Grajales'1, Alejandra Buenrostro Silva² \\ y Penélope R. TÉllez-Rodríguez ${ }^{3}$
}

${ }^{1}$ Instituto de Recursos, Universidad del Mar, campus Puerto Escondido, Km 2.5, carretera a Sola de Vega, San Pedro Mixtepec 71980, Oaxaca, MÉXICO. e-mail: archosaurio@yahoo.com.mx 2 Instituto de Industrias, Universidad del Mar, campus Puerto Escondido, Km 2.5, carretera a Sola de Vega, San Pedro Mixtepec 71980, Oaxaca, MÉXICO. e-mail: sba_1575@yahoo.com.mx

3 Licenciatura en Biología Marina, Universidad del Mar campus Puerto Ángel. Ciudad Universitaria, Km. 1.5, Carr. Puerto Ángel-Zipolite, Puerto Ángel 70902,

Distrito de San Pedro Pochutla, Oaxaca, MÉXICO.

García-Grajales, J., A. Buenrostro Silva y P. R. Téllez-Rodríguez. 2009. Variación de patrón de escutelación nucal del cocodrilo americano (Crocodylus acutus Cuvier 1807) en La Ventanilla, Oaxaca, México. Acta Zoológica Mexicana (n. s.), 25(2): 375-382.

RESUMEN. Se analizó el patrón de escutelación post-occipital y nucal de 111 cocodrilos (Crocodylus acutus) en La Ventanilla, Oaxaca. Se obtuvieron 33 patrones de escutelación diferentes. El patrón más frecuente observado (31.5\%) fue la presencia de 4 escudos post-occipitales, la ausencia de 2 escudos nucales anteriores externos y la presencia de 2 escudos nucales posteriores (4-2EE-2) y en menor proporción (6.31\%) se registró el arreglo considerado típico para la especie. Se observó una diferencia significativa entre las frecuencias de patrones más comunes encontrados en este trabajo y de la misma manera se realizó la comparación del patrón de escutelación más frecuente registrado en este estudio con respecto a otros reportes para $C$. acutus.

Palabras clave: Crocodylus acutus, patrón de escutelación, morfología, osteodermos.

García-Grajales, J., A. Buenrostro Silva \& P. R. Téllez-Rodríguez. 2009. Variation of nucal scutellation pattern of American crocodile (Crocodylus acutus Cuvier 1807) in La Ventanilla, Oaxaca, Mexico. Acta Zoológica Mexicana (n. s.), 25(2): 375-382.

ABSTRACT. The post-occipital and nucal scutellation patterns of 111 crocodiles (Crocodylus acutus) from La Ventanilla, Oaxaca were analyzed. Thirty three different scutellation patterns were found. The most frequently observed pattern $(31.5 \%)$ was 4 post-occipital scales present 2 external previous nucal scales absent, and 2 posterior nucal scales present (4-2EE-2). The pattern considered typical for this species was observed only in $6.31 \%$ of individuals. There were differences between the frequency distribution of common patterns when compared to other studies on C. acutus.

Key words: Crocodylus acutus, scutellation pattern, morphology, scales.

Recibido: 2 /10/2008; aceptado: 17/03/2009. 
García-Grajales et al.: Variación en la escutelación nucal de Crocodylus acutus

\section{INTRODUCCIÓN}

El cocodrilo americano (Crocodylus acutus) es una de las especies de mayor distribución en el mundo (Thorbjarnarson 1989, Ernst et al. 1999). Su distribución general incluye las costas del océano Atlántico y el Pacífico de México, Centro América y el norte de Sudamérica, así como las islas caribeñas de Cuba, Jamaica, Haití y República Dominicana y la punta sur de Florida, E. U. A. (Ernst et al. 1999).

Existe poca información disponible sobre las características morfológicas de esta especie, muchas de las cuales provienen de especímenes preservados en museos o de individuos mantenidos en cautiverio. Aunque se ha reconocido la variabilidad geográfica de la morfología de C. acutus (Neill 1971, Brazaitis 1973, Ross \& Mayer 1983), no ha sido cuantificada adecuadamente (Seijas 2002).

El patrón de escutelación del cocodrilo americano ha sido descrito por Brazaitis (1973), Ross \& Mayer (1983) y Ross \& Ross (1987), aunque sólo los dos últimos aportan información detallada acerca del origen y número de organismos examinados (Seijas 2002). Garrick (1982) analizó la variación del patrón de escutelación postoccipital y nucal del cocodrilo americano de diferentes localidades y tipos de hábitat. Para el caso de México, algunos autores (Álvarez del Toro 1974, Sigler 1993, Sigler datos no publicados, Villegas datos no publicados) observaron que esta especie presenta variaciones individuales en el patrón de osteodermos cervicales (postoccipitales y nucales), pero no realizaron una cuantificación completa.

El objetivo del presente trabajo fue analizar la variación del patrón de escutelación post-occipital y nucal en una población del cocodrilo americano en el estado de Oaxaca.

\section{MATERIAL Y MÉTODOS}

El trabajo se realizó en la Unidad de Manejo y Conservación de la Vida Silvestre (UMA) La Ventanilla, municipio de Santa María Tonameca, Oaxaca (Fig. 1), de julio de 2007 a febrero de 2008. Se estudió un grupo de 111 organismos colectados en el medio natural en las primeras horas después de la eclosión y llevados a la UMA La Ventanilla, con la finalidad de mantenerlos un año en cautiverio y liberarlos un año después y así disminuir la depredación en sus etapas más vulnerables (GarcíaGrajales 2005). Cada individuo fue marcado con un patrón único de cortes en las quillas caudales (Chabreck 1965) para su identificación individual posterior. Se registró el patrón de osteodermos post-occipitales y nucales siguiendo la propuesta de Villegas (datos no publicados) que consiste en trazar un eje imaginario longitudinal que divide a la región nucal en dos secciones (izquierda y derecha) y una línea imaginaria transversal que divide a los osteodermos en post-occipitales y nucales, posteriormente se contabilizaron los botones osteodérmicos presentes en esa región, tomando al organismo de la región posterior a la anterior y con vista dorsal (Fig. 2). 


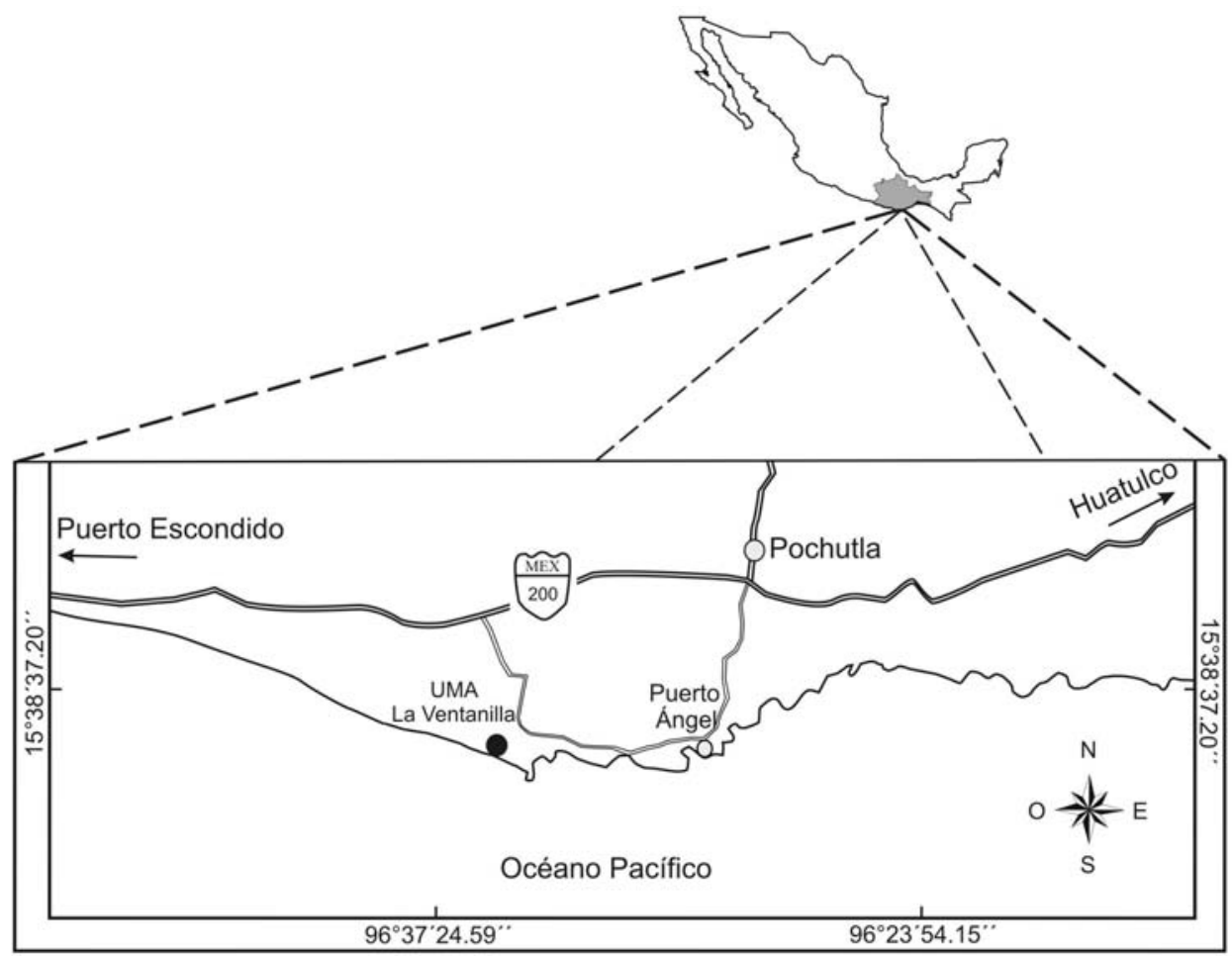

Figura 1. Ubicación de la Unidad de Manejo y Aprovechamiento Sustentable La Ventanilla, en la costa de Oaxaca, México.

A la agrupación de cuatro escudos post-occipitales (EPO) y seis escudos nucales (ENU) distribuidos en dos líneas (cuatro ENU anteriores y dos ENU posteriores), se le consideró como el patrón típico para la especie (Brazaitis 1973, Garrick 1982, Seijas 2002). Se utilizó una nomenclatura específica para determinar las variaciones del patrón de escutelación y en el que se describe la cantidad de escudos presentes por línea en cada región (EPO/ENU), tomando lectura de izquierda a derecha y marcando el sitio y lado donde hubiese ausencia de escudos con respecto al patrón típico (Fig. 2).

Se utilizó una prueba de $X^{2}$ (Zar 1999) para comparar la distribución de frecuencias entre los patrones de escutelación más frecuentes encontrados en este estudio. Adicionalmente, con esta misma prueba, se realizó una comparación para determinar si existían diferencias significativas entre el patrón más frecuente encontrado por Seijas (2002) y Villegas (datos no publicados) con respecto al patrón más frecuente observado por nosotros. 


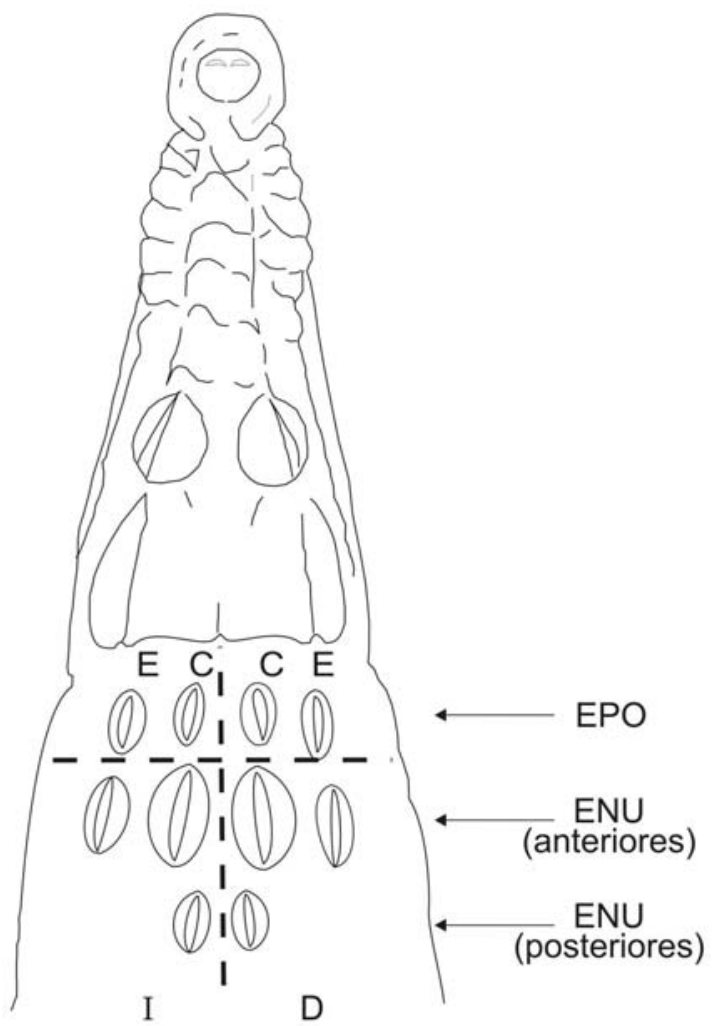

Figura 2. Representación del patrón de escutelación post-occipital (EPO) y escutelación nucal (ENU) del cocodrilo americano. La línea punteada horizontal separa los osteodermos postoccipitales de los nucales y la línea punteada vertical separa a los osteodermos en los planos izquierdo (I) y derecho (D).

\section{RESULTADOS}

Se registraron 33 patrones de escutelación diferentes, muchos de los cuales fueron poco frecuentes al estar representados por sólo uno o dos individuos (Cuadro 1). De los 111 organismos estudiados, 35 (31.5\%) presentaron el patrón 4-2EE-2 como el más frecuente, cuatro grupos de siete organismos $(6.31 \%$ por cada grupo) presentaron los patrones 2CC-2EE-2, 2CC-0-2, 4-2EE-1D y 4-4-2 (Fig. 3). Se observó diferencia significativa entre las frecuencias de patrones más comunes encontrados en este trabajo $\left(\mathrm{X}^{2}=44.22\right.$, g. $\left.1 .=2 \mathrm{P}<0.05\right)$. Cuando se comparó el patrón de escutelación más frecuente registrado en este estudio con cada uno de los patrones más comunes registrado en poblaciones de C. acutus de Venezuela y de la Bahía de Chetumal, México, se encontró diferencia estadísticamente significativa $\left(\mathrm{X}^{2}=101.47\right.$, g. $\left.1 .=2, \mathrm{P}<0.05\right)$. 
Cuadro 1. Patrones de escutelación registrados en el cocodrilo americano (Crocodylus acutus) en La Ventanilla, Oaxaca. EPO=Escudos Post-occipitales, ENUa=Escudo Nucales anteriores,

ENUp $=$ Escudos Nucales posteriores, $\mathrm{C}=$ Central, E=Externo, I=Izquierdo, $\mathrm{D}=$ Derecho.

\begin{tabular}{|c|c|c|c|c|}
\hline Patrón & EPO & ENUa & ENUp & Frecuencia \\
\hline 1 & 4 & $2 \mathrm{EE}$ & 2 & 35 \\
\hline 2 & $2 \mathrm{CC}$ & $2 \mathrm{EE}$ & 2 & 7 \\
\hline 3 & $2 \mathrm{CC}$ & 0 & 2 & 7 \\
\hline 4 & 4 & 2EE & $1 \mathrm{D}$ & 7 \\
\hline 5 & 4 & 4 & 2 & 7 \\
\hline 6 & $2 \mathrm{CC}$ & 2EE & $1 \mathrm{D}$ & 4 \\
\hline 7 & $2 \mathrm{CC}$ & 0 & 3IE & 3 \\
\hline 8 & 4 & $2 \mathrm{EE}$ & $1 \mathrm{I}$ & 3 \\
\hline 9 & 4 & 0 & 2 & 3 \\
\hline 10 & $3 \mathrm{IC}$ & 0 & 2 & 3 \\
\hline 11 & $3 \mathrm{DC}$ & $2 \mathrm{EE}$ & 2 & 3 \\
\hline 12 & $2 \mathrm{CC}$ & 4 & $1 \mathrm{D}$ & 2 \\
\hline 13 & $3 \mathrm{IC}$ & $2 \mathrm{EE}$ & $1 \mathrm{D}$ & 2 \\
\hline 14 & $2 \mathrm{CC}$ & 4 & 2 & 2 \\
\hline 15 & $2 \mathrm{CC}$ & $3 \mathrm{DE}$ & 2 & 2 \\
\hline 16 & $2 \mathrm{CC}$ & $2 \mathrm{CC}$ & $1 \mathrm{D}$ & 2 \\
\hline 17 & $1 \mathrm{CCDE}$ & 0 & 2 & 2 \\
\hline 18 & 0 & 4 & 2 & 2 \\
\hline 19 & $3 \mathrm{IC}$ & $2 \mathrm{EE}$ & 2 & 1 \\
\hline 20 & $3 \mathrm{IC}$ & $3 \mathrm{DE}$ & 2 & 1 \\
\hline 21 & $3 \mathrm{DC}$ & 4 & 2 & 1 \\
\hline 22 & $2 \mathrm{CC}$ & $2 \mathrm{EE}$ & $1 \mathrm{I}$ & 1 \\
\hline 23 & $2 \mathrm{CC}$ & $3 \mathrm{DC}$ & 2 & 1 \\
\hline 24 & $3 \mathrm{DC}$ & $2 \mathrm{EE}$ & $1 \mathrm{D}$ & 1 \\
\hline 25 & 4 & $3 \mathrm{IE}$ & 1I & 1 \\
\hline 26 & 4 & $3 \mathrm{DE}$ & 2 & 1 \\
\hline 27 & 4 & 4 & $1 \mathrm{D}$ & 1 \\
\hline 28 & $2 \mathrm{CC}$ & 4 & $1 \mathrm{I}$ & 1 \\
\hline 29 & 1CCDE & 0 & 3IE & 1 \\
\hline 30 & $2 \mathrm{CC}$ & 4 & 0 & 1 \\
\hline 31 & $2 \mathrm{CC}$ & $2 \mathrm{EE}$ & 0 & 1 \\
\hline 32 & 0 & $2 \mathrm{EE}$ & $1 \mathrm{D}$ & 1 \\
\hline 33 & 0 & 0 & 2 & 1 \\
\hline
\end{tabular}



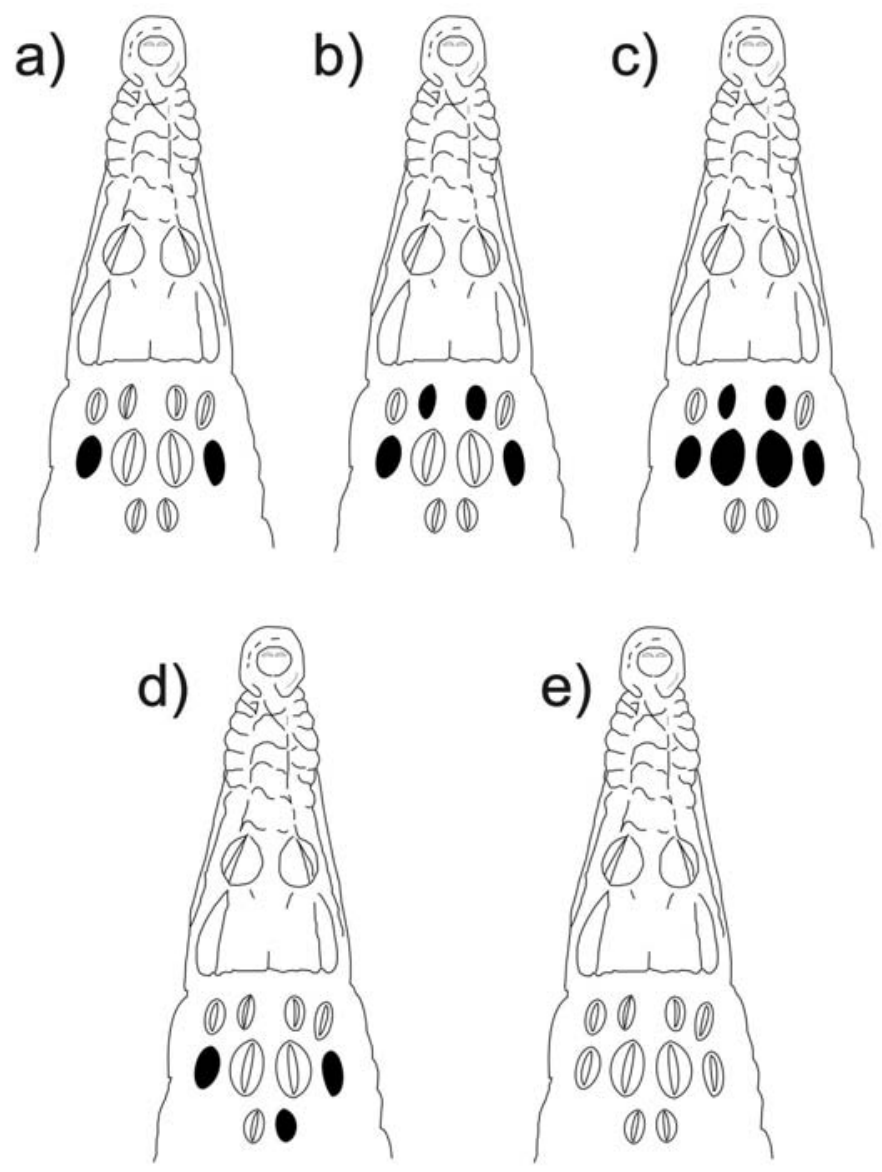

Figura 3. Algunos de los patrones encontrados en este estudio, a) Patrón 4-2EE-2, b) Patrón 2CC-2EE-2, c) Patrón 2CC-0-2, d) Patrón 4-2EE-1D, e) Patrón 4-4-2.

\section{DISCUSIÓN}

Aunque se ha reconocido la gran variabilidad del patrón de escutelación nucal que presenta el cocodrilo americano, mucha de la información disponible en la literatura no puede ser usada para estudios comparativos, debido a que no describen los patrones encontrados (Seijas 2002). El porcentaje de individuos con el patrón EPO/ENU típico (6.31\%) encontrado en este estudio fue mucho más bajo que los valores reportados por Ross \& Ross (1987) para México y Centroamérica (61.4\%), por Villegas (datos no publicados) para la Bahía de Chetumal, México (52.9\%) y por Seijas (2002) para varias localidades en Venezuela (77.6\%). 
Se sabe que el cocodrilo americano es la especie que presenta más variaciones en cuanto al número y disposición de los osteodermos cervicales (EPO/ENU). Villegas (datos no publicados) menciona que la disposición de osteodermos cervicales (EPO/ENU) encontrados en los cocodrilos de Bahía de Chetumal es variable llegándose a encontrar desde dos post-occipitales hasta cuatro y de dos hasta ocho, no obstante en los patrones detectados en el presente estudio se registraron individuos con ausencia de post-occipitales y como mínimo dos nucales. Brazaitis (1973) menciona que registró algunos organismos con sólo un osteodermo en los nucales, sin embargo esta condición no fue encontrada en los organismos bajo estudio en el presente trabajo y tampoco en los organismos analizados por Seijas (2002) en Venezuela.

La gran cantidad de patrones de escutelación diferentes (33) entre los individuos registrados en el estero La Ventanilla puede ser usada como evidencia de heterogamia. El desplazamiento de individuos es uno de los factores que trae consigo flujo genético entre las poblaciones (Porras-Murillo, 2004) y considerando la ubicación del Estero La Ventanilla y la cercanía con otros cuerpos de agua es muy probable que exista una alta interacción de individuos entre los cuerpos de agua. No debe descartarse el hecho de que las poblaciones de $C$. acutus en la costa de Oaxaca formen parte de una dinámica metapoblacional. La hipótesis de heterogamia por efecto de un arreglo metapoblacional podría ser probada mediante las técnicas modernas de la genética molecular.

AGRADECIMIENTOS. Los autores agradecen a la Universidad del Mar (UMAR) el financiamiento del trabajo (Clave interna: 2IR0705). Agradecemos la hospitalidad y facilidades prestadas para este estudio a la Sociedad Cooperativa de Servicios Ecoturísticos de La Ventanilla S. C. de R. L. de C. V. y por permitirnos realizar el trabajo en las instalaciones de la Unidad de Manejo para la Conservación de la Vida Silvestre SEMARNAT-UMA-EX-0009-OAXACA. Las observaciones de dos revisores anónimos contribuyeron a mejorar el contenido de este trabajo.

\section{LITERATURA CITADA}

Álvarez del Toro, M. 1974. Los Crocodylia de México. Instituto Mexicano de Recursos Naturales Renovables. México.

Brazaitis, P.E. 1973. The identification of living Crocodilians. Zoologica 58(3):59-101.

Chabreck, R. H. 1965. Methods of determining the size and composition of alligators populations in Louisiana. Proceeding 20th Annual Conference South eastern Association of game and fish commission. Pp. 47-50.

Ernst C. H., F. D. Ross \& C. A. Ross. 1999. Crocodylus acutus (Cuvier) American Crocodile. Catalogue of American Amphibians and Reptiles. 700: 1-17.

García-Grajales, J. 2005. Diseño de una estrategia de manejo del cocodrilo americano (Crocodylus acutus) en el estero La Ventanilla, Oaxaca, México. Tesis de Maestría. Instituto de Ecología A. C. Xalapa, Veracruz, México. 
García-Grajales et al.: Variación en la escutelación nucal de Crocodylus acutus

Garrick, L.D. 1982. Variation in postoccipital and nuchal scale patterns of American Crocodiles (Crocodylus acutus). American Zoologist 22(4):906.

Neill, W.T.1971. The last of the ruling reptiles. Columbia University Press. New York.

Porras-Murillo, L. P. 2004. Situación actual del cocodrilo Americano (Crocodylus acutus) en los ríos Jesús María, Tárcoles y Tusubres. Tesis de Maestría, Universidad Nacional Heredia, Costa Rica.

Ross, C. A. \& F. D. Ross. 1987. Identify of Crocodylus mexicanus Bocourt, 1869 (Reptilia:Crocodylidae). Proceedings of the Biological Society of Washington 100(4):713-716.

Ross, F. D. \& G. C. Mayer. 1983. On the dorsal armor of the Crocodilia. Pp. 305-301. In: A.G. Rhodin and K. Miyata (Eds). Advances in Herpetology and evolutionary biology-essays in honor of Ernest E. Williams. Harvard University, Cambridge.

Seijas, E. A. 2002. Scale patterns of American crocodiles (Crocodylus acutus) from several Venezuelan localities. Revista Unellez de Ciencia y Tecnología 20:118-134.

Sigler, L. 1993. Notas para la identificación, manejo y medidas para incrementar la población de cocodrilianos mexicanos en los zoológicos. Pp. 134-136. In: Anónimo. Memorias del IV Congreso de la Asociación Latinoamericana de Parques, Zoológicos y afines. Puebla, Puebla.

Thorbjarnarson, J. 1989. Ecology of the American crocodile (Crocodylus acutus). Pp. 228- 258. In: P. M. Hall (ed). Crocodiles: Their ecology, management and conservation. IUCN- The World Conservation Union Publications, Gland, Switzerland.

Zar, J.H. 1999. Biostatistical Analysis. 4th ed. Prentice Hall. E.U.A. 